\title{
Circuit
}

Musiques contemporaines

\section{Introduction. De l'appropriation au dialogue : des rencontres « transtraditionnelles » et leurs modalités}

\section{Sandeep Bhagwati et Jonathan Goldman}

Volume 28, numéro 1, 2018

De nouvelles racines pour une musique nouvelle ? Perspectives transtraditionnelles et transculturelles

URI : https://id.erudit.org/iderudit/1044371ar

DOI : https://doi.org/10.7202/1044371ar

Aller au sommaire du numéro

Éditeur(s)

Circuit, musiques contemporaines

ISSN

1183-1693 (imprimé)

1488-9692 (numérique)

Découvrir la revue

Citer ce document

Bhagwati, S. \& Goldman, J. (2018). Introduction. De l'appropriation au dialogue : des rencontres « transtraditionnelles » et leurs modalités. Circuit, 28(1), 9-14. https://doi.org/10.7202/1044371ar 


\section{Introduction. De l'appropriation au dialogue: des rencontres «transtraditionnelles » et leurs modalités}

Sandeep Bhagwati et Jonathan Goldman, traduit de l'anglais par Alice Boccara ${ }^{1}$

Les rencontres avec la musique (plutôt qu'avec des musiciens) d'autres traditions ont été capitales, et même décisives, pour la musique européenne de concert des cent dernières années. Entre les années 1890 et 1930, le fait de composer dans un contexte occidental a changé si fondamentalement de caractère qu'aujourd'hui encore, nombreux sont les auditeurs de musique classique qui considèrent les inventions musicales qui viennent après Brahms et postérieures à 1900 comme incompréhensibles, bizarres, voire perverses. Ces mêmes années ont aussi vu les premières rencontres des compositeurs avec la réalité sonore de nombreuses traditions musicales des lointains territoires et colonies de l'Europe. À l'époque déjà, de telles rencontres étaient pour la plupart médiatisées, tant par les expositions universelles et impériales que par le phonographe nouvellement inventé. Bien qu'il ne s'agisse certainement pas d'un simple cas où la coïncidence vaudrait corrélation, il pourrait être intéressant d'envisager la possibilité que la volonté et la force transformatrice des avant-gardes musicales du $\mathrm{xx}^{\mathrm{e}}$ siècle dérivent en grande partie du choc enivrant infligé à leur sensibilité musicale par la rencontre avec ces autres musiques. Alors que les compositeurs se retrouvaient face à l'évidence tangible et vivante qu'il existait un «ailleurs» avec des traditions musicales parfaitement développées et aux fondements théoriques et esthétiques totalement différents, ils en sont venus à la conclusion qu'une musique savante d'un genre nouveau n'était pas qu'une simple possibilité. S'il existait vraiment d'autres communautés qui écoutaient et appréciaient une musique que la théorie musicale européenne était totalement incapable d'expliquer, qui sait quoi d'autre encore serait possible?

\author{
1. La version originale anglaise de \\ cette introduction est disponible sur \\ le site internet de la revue. Visitez le \\ www.revuecircuit.ca/web/ (consulté le

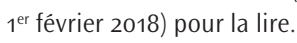


2. Terme inapproprié s'il en est (on peut rappeler à ce propos une anecdote concernant la visite nonannoncée que Barack Obama avait rendue en avril 2017 à l'Université de Chicago dans un cours de musique; et où, lorsque le professeur avait parlé de la Western art music, Obama, cet ancien leader du monde occidental à moitié kenyan et élevé en Indonésie, avait levé sa main et demandé innocemment ou sciemment - ce que ce terme signifiait.

3. Les instruments à cordes frottées (par un archet) ont été introduits en Europe par les cultures nomades d'Asie centrale, les instruments à anche par les cultures pastorales, et la plupart des instruments à percussion (qui ont été largement introduits en Europe au $\mathrm{xx}^{\mathrm{e}}$ siècle seulement) sont des instruments récemment assimilés venus d'Afrique, d'Asie de l'Est et d'Amérique latine. Seuls les cuivres, les claviers et les harpes semblent avoir évolué en Europe.

4. Anthony Tommasini, "Trump Is Wrong if He Thinks Symphonies Are Superior," New York Times, 30 juillet 2017, https://www.nytimes. com/2017/07/30/arts/music/trumpclassical-music.html?_r=o (consulté le 22 novembre 2017).

5. Voir le Glossaire proposé à même ce 10
Soulignons le fait que de telles rencontres ont eu lieu sur un terrain fertile: la pratique musicale européenne a toujours été le résultat de processus profondément syncrétiques et hybrides - et l'on pourrait même avancer l'idée que cette disposition à intégrer d'autres idées musicales, d'autres esthétiques et d'autres techniques est l'une des caractéristiques essentielles de la pratique musicale européenne. La musique européenne a toujours été une affaire passablement multiculturelle - il n'y a qu'à voir la façon dont les styles français, italien et allemand (et, occasionnellement, des orchestres de janissaires turcs...) ont fusionné ensemble pour former ce que nous désignons par l'expression anglaise Western art music ${ }^{2}$. La musique de concert à l'européenne a toujours été le résultat d'une absorption et d'une hybridation de traditions, qu'elles soient internes (pensons à ces compositeurs «nationalistes » du XIXe siècle qui se sont passionnés pour les musiques populaires de leur terre natale), ou externes (par exemple la passion de Dvořák pour la musique amérindienne, ou encore la rencontre de Gilles Tremblay et d'autres de ses contemporains québécois avec le gamelan, relatée par Jonathan Goldman dans ce numéro). En un sens, la musique de concert européenne a décrété sa propre expansion en tant que première tradition musicale d'envergure véritablement mondiale à travers sa logique interne d'appropriation et d'adaptation continuelle d'autres traditions - pas nécessairement en phase avec ses ambitions coloniales grandissantes, mais sous-tendue de toutes les façons par celles-ci, et qui trouva sa réalisation la plus concrète dans l'inclusion par des orchestres symphoniques contemporains de tant d'instruments d'origine extra-européenne'. Sachant cela, il est perturbant d'entendre l'actuel locataire de la Maison-Blanche, lors d'un discours donné à Varsovie le 6 juillet 2017, affirmer que «Nous écrivons des symphonies» comme s'il c'était là un trait essentiel séparant «l'Occident» du « reste » - et non un exemple de plus du syncrétisme identitaire tous azimuts de la culture occidentale ${ }^{4}$.

Si la logique de la musique occidentale a été celle d'une continuelle absorption et d'une hybridation de traditions, bien d'autres traditions musicales se sont consciemment positionnées en opposition à une telle gloutonnerie esthétique. Aussi bien pour des raisons de marchandisation que de défense culturelle, tout au long du $\mathrm{xx}^{\mathrm{e}}$ siècle, les traditions musicales nonoccidentales ont eu tendance à mettre en avant leurs identités musicales/ culturelles comme une alternative à la musique occidentale, s'affichant comme bien distinctes de celle-ci. Les compositeurs eurologiques ${ }^{5}$, jusqu'à la fin des années 1960, ne se sont penchés sur les théories et les sonorités issues de traditions musicales non eurologiques qu'en tant que catalyseurs de nouveaux langages musicaux ou comme simples boîtes à outils, et n'ont porté 
aucune attention particulière aux musiciens et au contexte social de chacun de ces systèmes musicaux. Conséquemment, des deux côtés, les nouvelles sensibilités postcoloniales se sont immédiatement emparées de cette conception identitaire de la musique traditionnelle. Le fait d'écouter des musiciens et de la musique issue de traditions non eurologiques est devenu un aspect important de la contre-culture européenne et nord-américaine, une façon d'exprimer clairement son mécontentement quant à l'ordre musical établi, au point où, au cours des années 1960 et 1970, ces musiques ont fini par effectivement devenir les alter ego de la musique occidentale. Bien sûr, le rôle joué par ces musiques alter ego, c'est-à-dire celui de servir de contre-modèle esthétique dans l'imaginaire occidental, impliquait que celles-ci soient protégées de la «contamination» des pratiques musicales, des théories et des réflexions contemporaines occidentales, la dichotomie ne pouvant marcher qu'entre deux choses « clairement» opposées. Ainsi, les besoins intellectuels des musiciens occidentaux anticonformistes ne pouvaient être satisfaits que par des traditions dites «authentiques» qui, d'une certaine façon, étaient aussi exemptes de l'historicisme des traditions esthétiques occidentales. Il n'est pas étonnant que les traditions musicales asiatiques, particulièrement, aient souvent été considérées comme des exemples vivants d'une musique venue de temps immémoriaux. En Asie, de nombreuses communautés ayant récemment retrouvé leur indépendance ont accepté cette invitation flatteuse à réaffirmer leur préséance culturelle sur leurs colonisateurs de jadis. Les deux camps se sont ainsi entendus sur une étrange division du monde entre une scène musicale eurologique en perpétuelle évolution, et d'autres traditions authentiques, éternelles, statiques...

Dans un même temps, la présence toujours grandissante de musiciens non occidentaux sur les scènes occidentales et les tournées mondiales de musiciens occidentaux tout au long du $\mathrm{Xx}^{\mathrm{e}}$ siècle ont créé des occasions pour les musiciens d'interagir directement entre eux, sans devoir recourir à la théorie ni passer par la médiation permise par la reproduction mécanique. La fin du $\mathrm{Xx}^{\mathrm{e}}$ siècle a vu naître de nombreuses tentatives de réunir des musiciens de différentes traditions culturelles (plutôt que seulement la musique en l'absence de ses praticiens originaux) afin qu'ils jouent ensemble, dans l'idée de permettre des échanges culturels bien-intentionnés ${ }^{6}$. Le moins qu’on puisse dire, c'est que, d'un point de vue musical, la plupart de ces rencontres ont été décevantes. Jusqu'à ce jour, si la majorité écrasante des rencontres musicales interculturelles ne mena pas simplement à l'écriture de partitions eurologiques qui utilisent les sonorités d'instruments extra-occidentaux sans aucune prise en considération de la tradition musicale de leurs origines, elle
6. Bien entendu, on sait à présent que nombre de rencontres Est-Ouest, particulièrement avec des musiciens indiens (par exemple la fameuse rencontre entre Ravi Shankar et Yehudi Menuhin), étaient organisées et chaudement encouragées par le "Congrès pour la liberté culturelle" financé par la CIA, avec le but de contrer l'œcuménisme culturel russe des Soviétiques avec ses adaptations orchestrales embourgeoisées et institutionnalisées de musiques traditionnelles populaires - dans le cadre d'un effort soutenu mené dans le but de rallier l'Inde nonalignée au camp occidental, au moins culturellement. https://www.hkw. de/en/programm/projekte/2017/ parapolitics/parapolitik_mehr_ zum_projekt/parapolitik_mehr.php (consulté le 20 décembre 2017). 
7. Les projets musicaux dépassant les frontières de leur propre tradition sont souvent dénigrés sous prétexte qu'ils n'appartiennent plus au royaume de la vraie musique, ou qu'ils ont "trahi" leur tradition d'origine. C'est aussi une suspicion assez répandue auprès des musiciens occidentaux et souvent appliquée aux musiciens qui osent expérimenter la transtraditionnalité. Ici, le concept d'une tradition "authentique" et "profonde" sert de cadre par défaut, selon lequel toutes les expérimentations transtraditionnelles sont considérées comme des entreprises intrinsèquement superficielles et trompeuses.

8. Bhagwati préfère le terme "intertraditionnel " plutôt que le plus commun « interculturel" pour indiquer que de telles rencontres et collaborations peuvent aussi se produire au sein de deux traditions appartenant à une même culture, comme par exemple entre le chant tyrolien (yodel) et la musique symphonique ou encore entre la musique hindoustanie et la musique carnatique, etc. Les problèmes peuvent être d'échelles différentes, mais sont de même nature. reposa néanmoins largement sur une attitude naïve d'improvisation de type jam session - l'équivalent en musique d'une poignée de main amicale et sans conséquences. Il n'est alors pas étonnant que ces projets se soient souvent contentés d'architectures structurelles, dramaturgiques, sonores et conceptuelles simplistes et éculées -, souscrivant fréquemment à un universalisme esthétique pseudo-égalitaire qui tend à faire abstraction, voire à nier activement l'existence de réelles différences et d'inégalités de pouvoir entre les intentionnalités musicales des musiciens, leurs possibilités et leurs contextes sociaux. Considérons, par exemple, le fait que de telles collaborations constituent, pour la plupart, des atouts pour le portfolio professionnel des musiciens occidentaux impliqués, tandis qu'elles tendent à avoir un impact neutre, voire négatif, sur la réputation de tout musicien extra-occidental participant au sein de sa «communauté» de pairs - particulièrement s'il vient d'une tradition qui souscrit à une définition auto-identitaire ${ }^{7}$ - ou bien qui, simplement, attribue à la nouveauté, à l'expérimentation ou à l'exploration une moindre valeur esthétique et morale intrinsèque que ne le fait la tradition eurologique. Les critiques, les musicologues, les musiciens et les compositeurs ont souvent été sceptiques quant à ces tentatives intertraditionnelles en musique. Ils les considèrent souvent comme musicalement et esthétiquement insatisfaisantes, en grande partie parce que de telles rencontres, même si elles sont plus équitables en termes d'impact sur la réputation des musiciens, semblent être délimitées par les intentions musicales eurologiques: on considère que les musiciens issus d'autres traditions du monde capitulent devant, ou bien flattent les goûts «classiques» et exotiques des publics occidentaux, sans qu'il n'y ait quasiment aucun changement requis de la part de l'orchestre en ce qui a trait à l'accordage, à l'improvisation, au placement du public, à la dramaturgie musicale, au cadre social, etc.

Le compositeur et titulaire de la chaire de recherche canadienne «Inter-X Arts», Sandeep Bhagwati, dirige le projet de recherche «Réassembler les extraits musicaux / Re-Assembling Musical Extracts » (financement du FQRSC, 2014-2017) qui a cherché à repenser les esthétiques de ce qu'il appelle les rencontres «intertraditionnelles» (intertraditional encounters) ${ }^{8}$ en travaillant au sein de deux projets collaboratifs de co-création, l'un avec un groupe d'interprètes issus de traditions musicales multiples, basé à Berlin, et l'autre à Montréal, les deux ensembles étant formés de musiciens urbains à l'aise avec des traditions savantes et improvisées, occidentales et non occidentales. Le point de départ de ce numéro de Circuit, c'est ce projet «Réassembler les extraits musicaux». De manière complémentaire, la section «Enquête » de ce numéro étudie les dynamiques de deux groupes musicaux à travers 
l'observation participative et les entretiens semi-dirigés menés par Deniza Popova et Julie Delisle.

Bien entendu, l'ensemble de Berlin (Extrakte Ensemble), l'ensemble de Montréal (Sound of Montréal) et la dernière génération d'orchestres européens interculturels décrits dans ce numéro par Katja Heldt et par l'un des plus éminents praticiens du genre, Stefan Östersiö, directeur artistique de l'ensemble interculturel suédois The Six Tones, de même que le nombre grandissant d'ensembles interculturels canadiens décrits ici par Jeremy Strachan, sont des exercices d'hybridation et, par là-même, véhiculent tous les risques bien connus qui sont associés aux formes hybrides, en particulier ceux qui touchent aux dynamiques géopolitiques contemporaines et à l'héritage du colonialisme. Il est vrai que certains théoriciens postcoloniaux, et en particulier Homi Bhabha, ont célébré l'hybridation comme une valeur positive, selon ce que celui-ci appelle un «tiers-espace» qui «permet à d'autres positions d'émerger » en déplaçant «les histoires qui [les] constituent et [en mettant] en place de nouvelles structures d'autorité, de nouvelles initiatives politiques, qui sont comprises de façon inadéquate comme des idées reçues». Pour Bhabha, «le processus d'hybridation culturelle donne naissance à quelque chose de différent, de nouveau et de méconnaissable, un nouvel espace de signification et de représentation ${ }^{9}$ ». En même temps, beaucoup, voire la plupart des spécialistes contemporains de l'hybridation sont conscients des écueils potentiels des projets artistiques fondés sur l'hybridation interculturelle. Sarah Weiss, par exemple, exprime cette préoccupation lorsqu'elle décrit le spectacle I La Galigo du metteur en scène Robert Wilson, inspirée par les légendes du peuple bugis du Sulawesi du Sud (Célèbes), en Indonésie:

Selon la perspective, le spectacle $[. .$.$] peut apparaître [\ldots]$ comme une extension du processus colonial occidental; un vol malhonnête et symbolique de la culture bugis par les Indonésiens et «l'Occident»; une simplification appauvrie du complexe récit bugis; une représentation authentique des différents aspects de la culture bugis; un vivant exemple des possibilités et du potentiel de l'échange interculturel à différents niveaux ${ }^{10}$.

Ce malaise est soulevé dans plusieurs des contributions qui composent l'édition 2016 du Routledge International Handbook of Intercultural Arts Research ${ }^{11}$. Il n'est pas inconcevable que chacun de ces jugements puisse être vrai, mais réduire les contours nuancés de l'interculturalité artistique à un jugement de valeur binaire et, partant, condamner au silence le débat universitaire, lequel ne peut commencer qu'à partir du moment où la nature du projet est bien comprise et donc documentée, est totalement contraire aux objectifs de cette édition de Circuit.
9. Cité in Timothy D. Taylor (2007), Beyond Exoticism: Western Music and the World, Durham, Duke University Press, p. 145.

10. Sarah Weiss (2008), "Permeable Boundaries: Hybridity, Music and the Reception of Robert Wilson's / La Galigo," Ethnomusicology, vol. 52, $\mathrm{n}^{\circ} 2$, p. 203-238, 204.

11. Pamela Burnard, Elizabeth Mackinlay et Kimberly Powell (éd.), Routledge International Handbook of Intercultural Arts Research, Londres, Routledge, 2016. 
Bien entendu, le modèle d'hybridation dans la forme qu'il a prise dans de nombreux projets musicaux interculturels au $\mathrm{XX}^{\mathrm{e}}$ siècle ne parvient pas à transcender le nationalisme, et, à vrai dire, le renforce, car ce modèle repose sur la conception selon laquelle la musique d'une culture vient se mélanger à une autre, combinant une essence $\mathrm{X}$ à une essence $\mathrm{Y}$. En conséquence, il n'est pas surprenant de constater la persistance tenace et désolante de catégories enracinées dans le modèle de l'État-nation qui infuse encore le discours et la prise de décision des administrateurs et des directeurs artistiques du monde de la musique savante (qu'il s'agisse de classifier des participants à un atelier d'un compositeur contemporain selon leur pays d'origine, ou bien d'intituler des concerts philharmoniques «Le destin slave»). À cet égard, Jin-Ah Kim offre une appréciation particulièrement opportune des façons dont le compositeur Sandeep Bhagwati approche la musique intertraditionnelle, à travers son exploration de longue date de la «comprovisation», une forme hybride en soi qui brouille les frontières entre la composition fondée sur la partition et celle fondée sur l'improvisation.

Inévitablement, les contributeurs de ce numéro soulèvent plus de questions qu'ils n'en résolvent, mais même s'ils parviennent à offrir un modeste aperçu de ce qui existe «ailleurs», nous aurons réussi à faire avancer la discussion concernant la création musicale contemporaine dans une direction nouvelle, et, espérons-le, fructueuse.

Bonne lecture!

Montréal, décembre 2017 